\title{
A novel SPECT camera for molecular imaging of the prostate
}

\author{
Alan Cebula*a , David Gilland ${ }^{\mathrm{a}}$, Li-Ming Su ${ }^{\mathrm{b}}$, Douglass Wagenaar ${ }^{\mathrm{c}}$, Amir Bahadori $^{\mathrm{a}}$ \\ ${ }^{\mathrm{a} J}$. Crayton Pruitt Family Dept. of Biomedical Engineering, University of Florida, \\ 202 NSC, Gainesville, FL, USA 32611-8300; \\ ${ }^{b}$ Dept. of Urology, University of Florida, 2000 Sw Archer Rd, Gainesville, FL, USA, 32610; \\ ${ }^{\circ}$ Children's Hospital of Los Angeles, 4650 Sunset Blvd, Los Angeles, CA,USA 90023
}

\begin{abstract}
The objective of this work is to develop an improved SPECT camera for dedicated prostate imaging. Complementing the recent advancements in agents for molecular prostate imaging, this device has the potential to assist in distinguishing benign from aggressive cancers, to improve site-specific localization of cancer, to improve accuracy of needle-guided prostate biopsy of cancer sites, and to aid in focal therapy procedures such as cryotherapy and radiation. Theoretical calculations show that the spatial resolution/detection sensitivity of the proposed SPECT camera can rival or exceed 3D PET and further signal-to-noise advantage is attained with the better energy resolution of the CZT modules. Based on photon transport simulation studies, the system has a reconstructed spatial resolution of $4.8 \mathrm{~mm}$ with a sensitivity of 0.0001. Reconstruction of a simulated prostate distribution demonstrates the focal imaging capability of the system.
\end{abstract}

Keywords: Single Photon Emission Computed Tomography (SPECT), Prostate Imaging, Cadmium Zinc Telluride (CZT)

\section{INTRODUCTION}

Prostate cancer is the most common solid organ cancer in men in the United States with over 217,730 new cases and close to 32,000 deaths per year based upon 2010 statistics by the American Cancer Society. ${ }^{1}$ It is estimated that 1 man in 6 will be diagnosed with prostate cancer during his lifetime with 1 man in 36 dying of the disease. The diagnosis of prostate cancer has historically relied upon ultrasound-guided prostate needle biopsy. Despite advances in more extensive prostate needle biopsies schemes, approximately $30 \%$ of cancers may be missed on initial biopsy, resulting in underdiagnosis and staging of cancer that is truly present. ${ }^{2}$ As a result, there has been active investigation into alternative imaging methods for the diagnosis of prostate cancer including CT, multiparametric functional MRI (T2 MR, MR spectroscopy, dynamic contrast enhanced MR, diffusion weighted MR), ultrasound, PET, and SPECT. ${ }^{3-6}$ In addition to more accurate, early stage detection, other critical roles for prostate cancer imaging include differentiating aggressive from benign cancers, pinpointing micro-disease location to guide biopsy and enable focal therapy, and assisting in prostate surgery particularly at the prostate margins. ${ }^{3,7,8}$ At the margins critical surgical decisions must be made between removing adequate tissue so that residual cancer is not left behind but not so much tissue as to cause incontinence and impotence.

Active investigation is underway toward developing better PET and SPECT agents for molecular imaging of prostate cancer. In the past, the major drawback of these agents, such as ${ }^{18} \mathrm{~F}-\mathrm{FDG}$ and ${ }^{11} \mathrm{C}$-choline, has been the high bladder activity, which obscures visualization of the adjacent prostate gland, and low tumor uptake due to the relative low glucose metabolism of prostate cancer. ${ }^{9,10}$ The new molecular imaging agents are being designed for improved tumor uptake and decreased non-target tissue uptake. Specifically, molecular imaging agents targeting prostate-specific membrane antigen (PSMA) are currently being investigated. ${ }^{11-20}$ Already, SPECT radionuclides have been labeled with small molecule PSMA inhibitors. ${ }^{15,21-23}$ PSMA is used as a marker for androgen-independent disease which may

*acebula@ufl.edu

Medical Applications of Radiation Detectors, edited by H. Bradford Barber, Hans Roehrig,

Douglas J. Wagenaar, Proc. of SPIE Vol. 8143, 814305 - () 2011 SPIE

CCC code: $0277-786 X / 11 / \$ 18 \cdot$ doi: $10.1117 / 12.896235$

Proc. of SPIE Vol. $8143814305-1$ 
distinguish between indolent and aggressive tumors. Preclinical models and phase 1 clinical results show high tumor to normal tissue uptake ratio for a low molecular weight, ${ }^{99 \mathrm{~m}} \mathrm{Tc}$ labeled agent. ${ }^{22}$ Five ${ }^{99 \mathrm{~m}} \mathrm{Tc}$-labeled analogs provided clear tumor delineation in vivo, with low background at 4 hours post-injection. ${ }^{15}$ Remarkably, some first generation ligands have demonstrated nearly a 50 to 1 target to non-target ratio in preclinical animal models. ${ }^{15}$

To complement the effort toward developing better SPECT agents for prostate cancer, there is a need for improved SPECT imaging methods and instrumentation. For prostate imaging, the drawback of conventional SPECT methods-in which a camera equipped with a parallel hole collimator and sodium iodide (NaI) detector rotates around the pelvis - is that throughout the acquisition the prostate gland maintains a relatively large distance to the camera with a substantial amount of intervening tissue. The large distance results in poor spatial resolution, and the intervening tissue increases photon attenuation and scatter effects. The scatter effects are compounded by the proximity of the bladder to the prostate gland and the substantial radionuclide level that can be present in the bladder.

The goal of this work is to develop a better SPECT camera for molecular imaging of prostate cancer. The novel camera design proposed here has the potential to overcome the drawbacks of conventional SPECT methods in prostate imaging. The stationary camera can be positioned close to the prostate to achieve high spatial resolution, reduced intervening tissue, and mechanical stability. The collimator is a multi-pinhole design, which offers better spatial resolution and sensitivity compared to traditional parallel hole collimators for short source-to-collimator distances. Historically, multipinhole collimators have been used in pre-clinical imaging systems, ${ }^{24-36}$ and more recently in a commercial, dedicated cardiac system. ${ }^{37}$ Finally, advanced detector technology incorporating cadmium zinc telluride (CZT) will be utilized to achieve high energy resolution, reduced scatter effects and improved signal-to-noise ratio, which can mitigate the effects of radionuclide present in the adjacent bladder. The combination of both CZT detectors and multiple pinholes has been developed for a small animal imaging system. ${ }^{38}$

This paper describes the design of the dedicated SPECT prostate imager and evaluates the system performance using photon transport simulations. The basic system performance characteristics including spatial resolution and sensitivity are measured from point sources in air and in a water phantom. In addition, reconstructed image quality is assessed using an anthropomorphic phantom that models realistic prostate and bladder source distributions and patient attenuation and scatter characteristics.

\section{METHODS AND MATERIALS}

\subsection{Camera design}

The large source-to-collimator distance using a parallel hole collimator results in low spatial resolution which can only be improved with a loss of sensitivity and increased image noise. The novel design of the dedicated SPECT prostate imager proposed here ("pSPECT") uses a morphology-based, saddle shaped collimator with multiple, focused pinholes to image the prostate and pelvic volumes of interest. Figure 1 shows a rendering of the collimator and detector geometry implementing multiple pinholes. The collimator is represented by the blue surface with the green cones showing the field of view (FOV) of three of the sixteen pinholes. The close proximity of the pinholes to the prostate will allow for better spatial resolution compared to conventional SPECT systems while maintaining high sensitivity. Furthermore, the stationary camera simplifies the mechanical aspects of the system and improves stability.

Manufacturing the body of the physical collimator will be accomplished using a cold casting technique with tungsten powder alloy. ${ }^{39}$ The cold casting process of collimator production has been previously implemented in small animal imaging systems. ${ }^{40}$ The ability of the powder and epoxy to conform to a mold allows for flexibility in collimator design. The molds are easily developed using 3D computer-aided design software and 3D printers in a rapid prototyping technique. Holes would be designed within the collimator body to allow for the placement of precision machined tungsten inserts for the pinholes. The contour of the pSPECT collimator will be a comfortable saddle shape and closely follow patient morphology in the region of the prostate. A range of collimators would be constructed to ensure patient comfort and optimal imaging. 

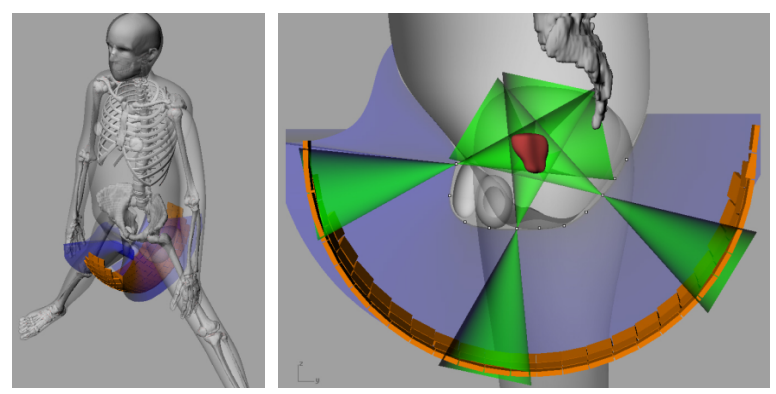

Figure 1. Dedicated prostate SPECT imager (“pSPECT”)

Another significant aspect of the proposed system is the use of compact, high energy resolution CZT detector technology. The CZT detectors have superior energy resolution compared to conventional NaI detector systems with $10 \%$ energy resolution. Energy resolutions as low as 5\% have been previously implemented in molecular breast imaging systems. ${ }^{41}$ Higher energy resolution reduces the effect of scatter and improves contrast-to-noise ratio. An example of a modular CZT detector manufactured by Gamma Medica, Inc. (GMI) ${ }^{42}$ is shown in Figure 2 . The detector module is 2.54 $\mathrm{cm}$ square with $1.6 \times 1.6 \times 5 \mathrm{~mm}$ pixels and can be tiled using flexible cables. Using a modular CZT detector allows for a non-coplanar detector design as shown by Figure 2. The pSPECT detectors are shown by the orange surface in Figure 1.
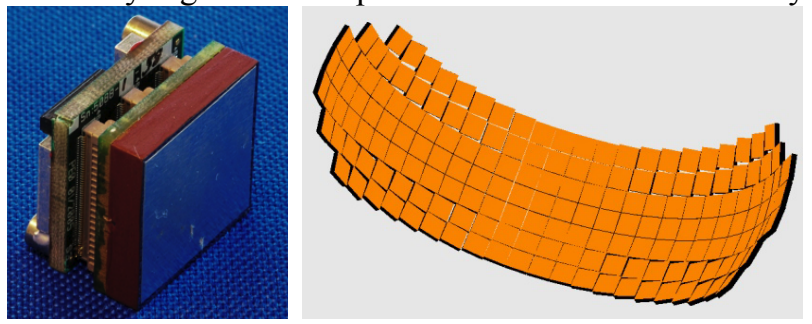

Figure 2. CZT detector module (GMI) and tiled imaging plane

The collimator-detector design was spatially modeled using non-uniform rational B-spline (NURBS) surfaces in Rhinoceros. $^{43}$ For the scaled, supine patient model, 6 pinholes covering approximately 130 degrees for a point in the center of the FOV were configured for imaging the prostate. The average distance from a point near the center of the prostate to each pinhole was $8.6 \mathrm{~cm}$. However, it is expected this distance would decrease for a patient sitting on the saddle due to body weight deforming tissues between the prostate and perineum. The array of detector modules (Fig. 2) was placed at a focal length of $14 \mathrm{~cm}$ for each pinhole. A pinhole acceptance angle of 36 degrees was used to maximize the FOV without multiplexing the six pinholes. This configuration provides a $5.8 \mathrm{~cm}$ FOV at a source to collimator distance of $8 \mathrm{~cm}$. Figure 3 shows the placement of the pinholes and tiled detector modules with respect to the prostate.

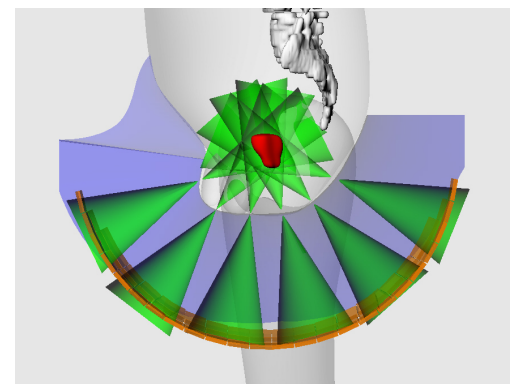

Figure 3. Scaled NURBS model showing six pinholes (green) configured to image the prostate

\subsection{Spatial Resolution and Sensitivity Analysis}

Predicted spatial resolution at the object plane was determined using the collimator and detector spatial resolution for a point source located near the center of the prostate. ${ }^{44}$ The intrinsic spatial resolution of the CZT detectors is $1.59 \mathrm{~mm}$ and pinhole spatial resolution can be determined by the formula ${ }^{45}$

$$
R_{c}=\frac{d_{e f f} \cdot(l+b)}{b}
$$


Using an effective pinhole diameter $d_{\text {eff }}=3.08 \mathrm{~mm}$, a focal length $l=14 \mathrm{~cm}$, and a source to collimator distance $b=8.6$ $\mathrm{cm}$, the expected spatial resolution of the system is $5 \mathrm{~mm}$. The predicted system sensitivity was determined by multiplying the geometric sensitivity by the number of pinholes and an exponential attenuation factor for $140 \mathrm{keV}$ photons in water. Geometric sensitivity can be computed using the formula ${ }^{45}$

$$
g=\frac{d_{e f f}^{2} \cdot \cos ^{3} \theta}{16 \cdot b^{2}}
$$

For a $\theta=0$ photon path angle, the predicted system sensitivity is 0.00013 . These predicted values likely underestimate the performance of the system because the prostate is expected to move closer to the perineum when the patient is in a sitting position. For an estimated source to collimator distance $b=5 \mathrm{~cm}$ and $5 \mathrm{~mm}$ spatial resolution, the pSPECT sensitivity is 0.001. The anticipated spatial resolution/sensitivity of pSPECT for focal prostate imaging is on par with current 3D PET systems. The same analysis applied to a 3D PET using the formula for sensitivity ${ }^{45}$ with an $85 \mathrm{~cm}$ diameter ring, $16 \mathrm{~cm}$ axial extent and $36 \mathrm{~cm}$ photon path length for $511 \mathrm{keV}$ photons in water results in a sensitivity of 0.0031 . A further advantage of the pSPECT system is the effect of the improved energy resolution of CZT, and the resulting reduction in detected scatter compared with PET.

The predicted spatial resolution/sensitivity performance of pSPECT far exceeds conventional SPECT for imaging the prostate. Using formulas for the spatial resolution and sensitivity for a parallel hole collimator ${ }^{45}$ and assuming a collimator hole length of $4 \mathrm{~cm}$, a hole diameter of $2 \mathrm{~mm}$, an object distance of $20 \mathrm{~cm}$, intrinsic spatial resolution of 3.5 $\mathrm{mm}$, attenuation path length of $16 \mathrm{~cm}$ of water $(140 \mathrm{keV})$, and a triple head camera, the predicted spatial resolution is $12.5 \mathrm{~mm}$ with a sensitivity of 0.000046 .

\subsection{Computational evaluation using Monte Carlo}

Patient and detector models (Fig. 3) were developed that can be readily incorporated into radiation transport code to assist in the system design and to investigate the effects of design parameters on reconstructed image quality. The patient was modeled using the UF Hybrid Adult Male Phantom (UFHADM). The UFHADM phantom uses a NURBSbased $^{46}$ representation of the patient (Fig. 4a) and has been used extensively in computational transport studies. ${ }^{47}$ Radioactivity uptake and linear attenuation coefficient values can be assigned to the individual organs of the phantom. Realistic representations of all major body organs including the prostate are incorporated into the phantom and the body habitus of the simulated patient can easily be varied. The body used for this simulation is 50 percentile in height and weight. The radiation transport code Monte Carlo N-Particle version 5 (MCNP5) ${ }^{48}$ was used to simulate projection images. The phantom was voxelized to a resolution of $2 \times 2 \times 2 \mathrm{~mm}$ (Fig. 4b) using an in-house MATLAB code and defined in MCNP5 using the lattice and universe cards. Each voxel value corresponded to the appropriate material number for each tissue and was defined using the fill card in MCNP5. The pSPECT camera, including the saddle-shaped multi-pinhole collimator and the CZT detector, were represented using standard surfaces within the MCNP5 code to compute the radiation transport to the detector (Fig. 4c). The collimator design being evaluated here includes six pinholes focused on the prostate. Collimator parameters from the spatial resolution and sensitivity analysis of section 2.2 will be used.

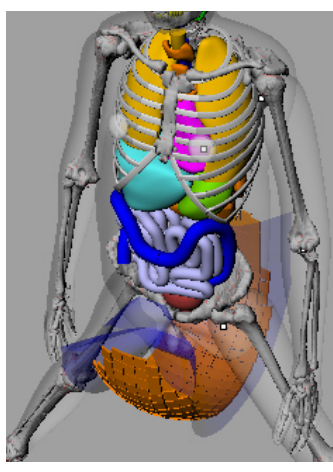

(a)

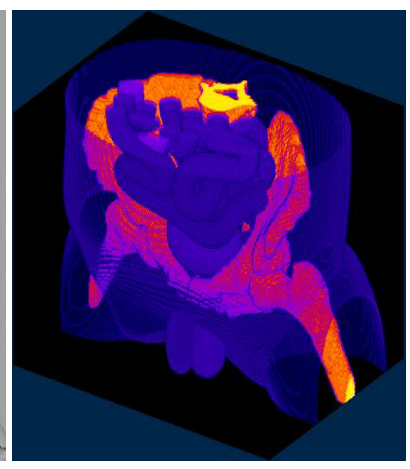

(b)

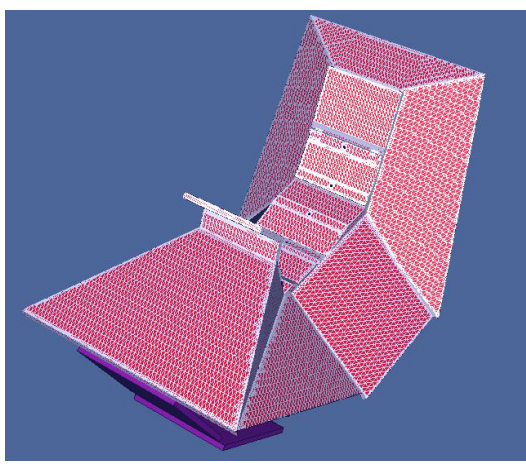

(c)

Figure 4. UFHADM phantom positioned on the pSPECT camera (a) and renderings of the voxel phantom (b) and saddle-shaped collimator (c) inputs for MCNP5 
Interactions within each detector were recorded using the PTRAC card in MCNP5. Since the PTRAC card is not compatible with multicore processing, the transport simulation was divided into two steps. On a computer cluster of 48 processors, photons were transported through the collimator to the face of each detector and recorded with the surface source write card. Then a subsequent serial run was carried out to simulate the transport of photons from the surface source through the detector. To reduce computation time, variance reduction was implemented using source biasing techniques for the multicore simulations. A MATLAB code was written to process the PTRAC output file and determine the location and amount of energy deposited in each pixel.

The processed PTRAC energy data represented a detector with perfect energy resolution. Detector energy resolution was modeled by randomly sampling each event from a probability distribution representing the energy response function of the detector. To simulate the asymmetric low energy tail of CZT, a combination of a truncated exponential and Gaussian was fit to a measured spectrum of a 4.8\% FWHM detector as shown in Figure 5. A photon event contributed to the projection image if the resampled energy was within a $+/-10 \%$ energy window. Sufficient detector events were generated to produce low noise projection images. To simulate realistic noise distributions, the projection images were scaled to account for source strength and acquisition time then Poisson noise was added. Reconstruction was carried out using an adaptation of the standard iterative maximum likelihood estimation maximization (MLEM) algorithm. The MLEM algorithm was written to allow for a general pinhole configuration. The system matrix modeled the effects of photon entry angle on sensitivity. The system matrix did not model attenuation or spatial resolution effects. A total of 500 iterations were used to ensure the convergence of the reconstruction. For the annulus and phantom distributions, post-reconstruction filtering was completed using a Hann filter with a cut-off of 0.5 cycles per pixel.

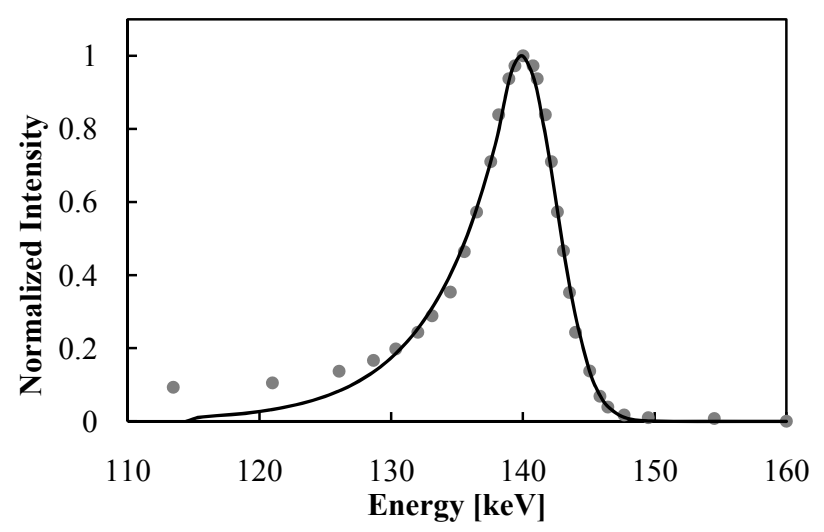

Figure 5. Photopeak response of CZT used for modeling energy resolution

System performance was evaluated for multiple source configurations as shown in Figure 6 for $140 \mathrm{keV}$ photons. To determine reconstructed spatial resolution and sensitivity, a point source in air was simulated (Fig. 6a) at the center of the FOV for all six pinholes. Figure $6 \mathrm{~b}$ shows the water phantom and point source geometry used for simulating the effects of scatter. Reconstructed image quality was also assessed for an annulus source with an outside diameter of $3 \mathrm{~cm}$ and thickness of $3 \mathrm{~mm}$ as shown in Figure 6c. Realistic, patient source distributions were modeled using the voxelized UFHADM phantom shown in Figure 6d. Uptake was modeled for the whole prostate, bladder, body, and a localized tumor. Figure 7 shows the location of the simulated $4 \mathrm{~mm}$ diameter tumor in the mid-peripheral zone. ${ }^{49}$

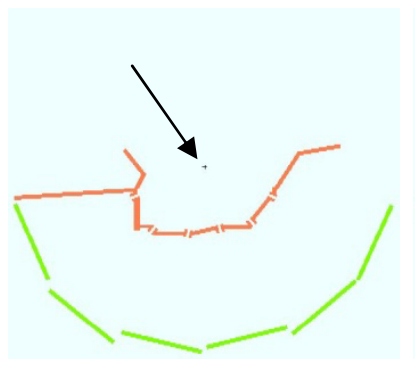

(a)

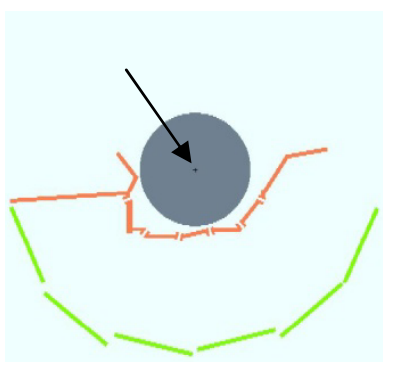

(b)

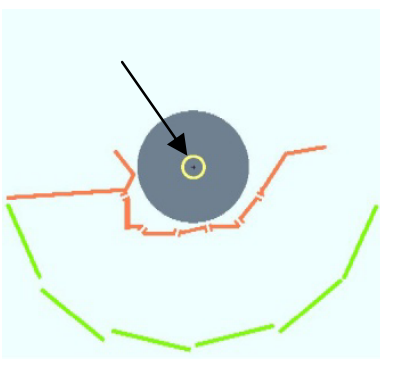

(c)

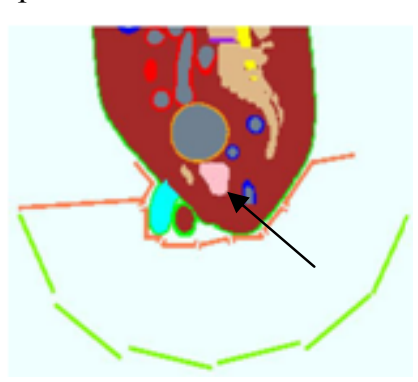

(d) 
Figure 6. Mid-sagittal slice of the MCNP5 geometry for each source configuration showing the collimator (orange), detectors (green), prostate (pink) within the voxelized phantom, and source location (arrow)
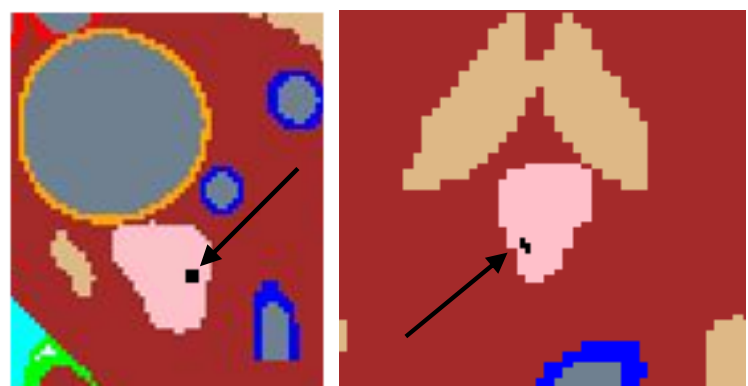

Figure 7. Sagittal and transaxial phantom images showing the location of localized tumor uptake (black) within the prostate (pink)

\section{RESULTS}

Figures 8 and 9 show reconstructed images and line profiles for a point source in air (Fig. 8) and a water phantom (Fig. 9). From the associated line profile, the spatial resolution of the system was calculated to be $4.7 \mathrm{~mm}$ for a point source in air and $4.8 \mathrm{~mm}$ for a point source in water. The reduced colormap window for the reconstructed point source in water (Fig. 9b) demonstrates the limited amount of out of source activity from scatter. For the point source in air, the sensitivity was calculated to be 0.0001 .
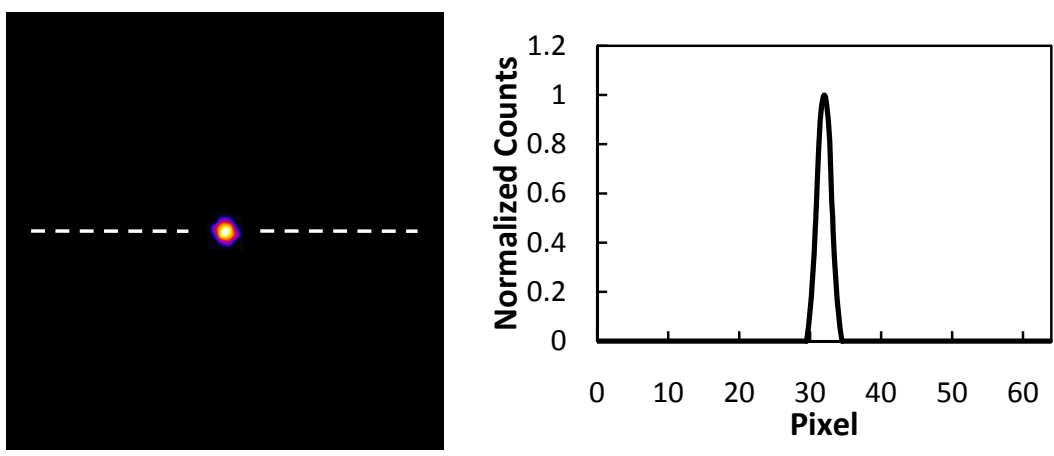

Figure 8. Reconstructed image and line profile for a point source in air

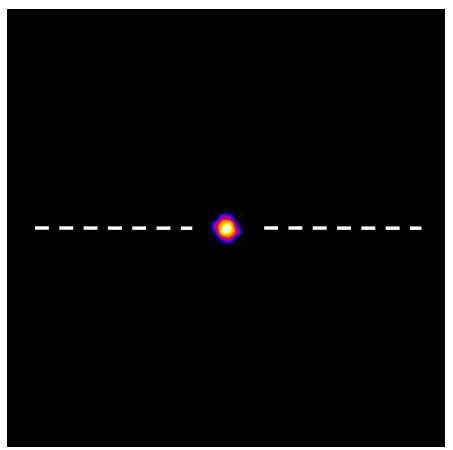

(a)

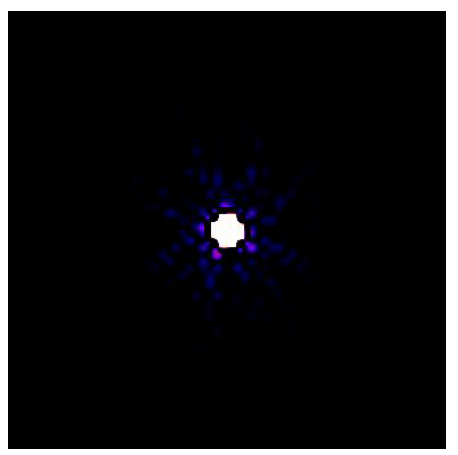

(b)

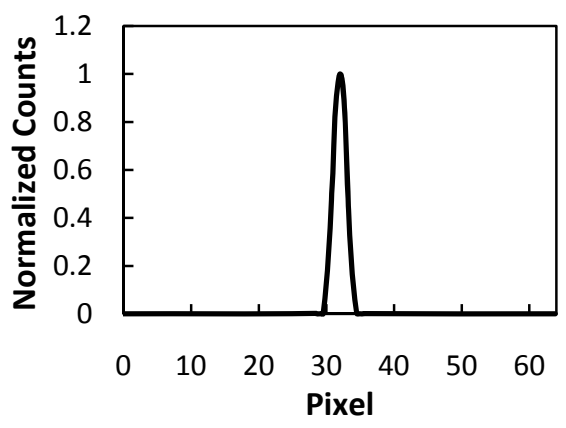

Pixel

Figure 9. Reconstructed point source in a water phantom with full color display window (a) and reduced window (b) showing out of source activity.

Reconstructed images of the annulus source are shown in Figure 10. For the six pinhole configuration, a slight artifact is evident corresponding to the base of the prostate (Fig. 10a). The artifact increases as the number of pinholes is reduced to five and four (Fig. 10b,c). Transaxial and sagittal images of a reconstructed, uniform prostate distribution are shown 
in Figure 11 for a prostate uptake of $200 \mu \mathrm{Ci}$ and an imaging time of 30 minutes. Focal imaging results of the simulated $4 \mathrm{~mm}$ diameter tumor are shown in Figure 12. The images represent an acquisition time of 30 minutes with a $30 \mathrm{mCi}$ injected activity. The uptake fraction was 0.1 for the bladder, 0.7 for the body, and 0.0067 for the prostate. This resulted in a bladder to prostate concentration of 1.22. The images show the response of the system for varying degrees of tumor to prostate $(\mathrm{T}: \mathrm{P})$ concentrations.

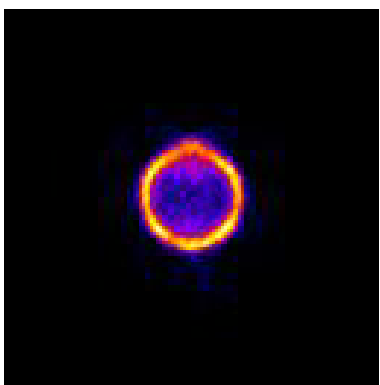

(a)

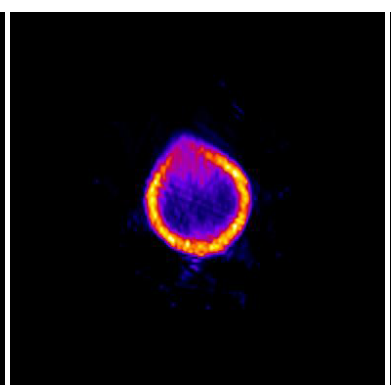

(b)

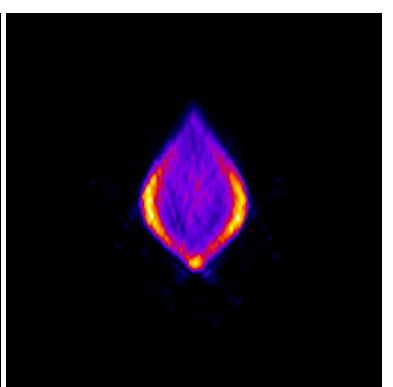

(c)

Figure 10. Sagittal slices of the reconstructed annulus source for the 6 (a), 5 (b), and 4 (c) pinhole configurations

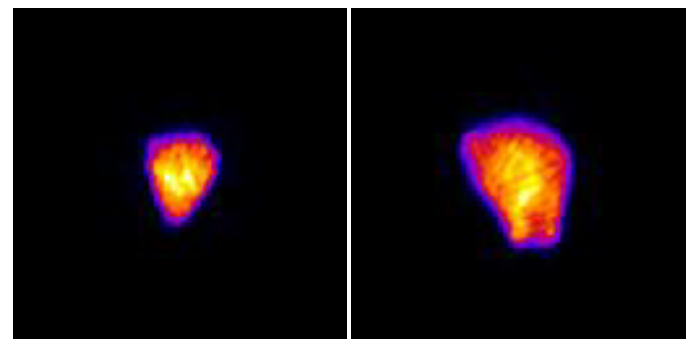

Figure 11. Transaxial (left) and sagittal (right) reconstructed images of a uniform prostate distribution

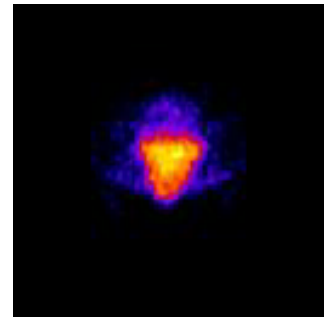

$\mathrm{T}: \mathrm{P}=1$

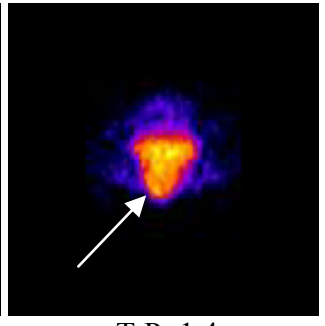

$\mathrm{T}: \mathrm{P}=1.4$

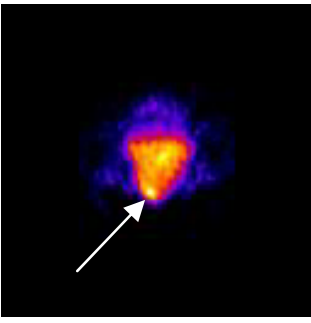

$\mathrm{T}: \mathrm{P}=2.8$

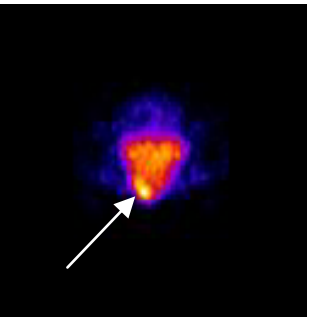

$\mathrm{T}: \mathrm{P}=3.8$

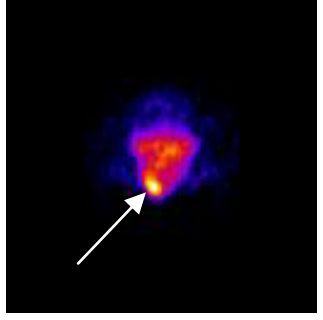

$\mathrm{T}: \mathrm{P}=5.7$

Figure 12. Transaxial images of uniform prostate, body, and bladder distributions with a localized tumor of located in the peripheral zone. The bladder to prostate concentration is 1.22 with varying levels of tumor to prostate $(\mathrm{T}: \mathrm{P})$ concentrations.

\section{DISCUSSION}

The predicted spatial resolution and sensitivity of the system agreed well with the calculated performance for the simulated point source in air. Based on the point source in a water phantom simulation, the high energy resolution of the CZT detectors limits the effects of scatter on reducing the reconstructed image quality. The annulus source reconstruction demonstrates how the limited angular coverage of the six pinhole design mainly affects image quality for points near the base of the prostate. Further reduction of the angular coverage would reduce the cost of the system, but at the expense of greatly reduced image quality. In contrast, an additional pinhole may alleviate the basal artifact.

Expansion of the system to perform nodal imaging and increase sensitivity could be achieved by increasing the number of pinholes. Adding additional pinholes for a given detector size leads to multiplexing and reduced spatial information of multiplexed events. Figure 12 shows the extent of multiplexing for a sixteen pinhole configuration. To limit multiplexing and maximize the fill factor of the detector array, a secondary shield could be used to form a square aperture response. This square aperture would affect the spatial resolution response of the system. The anisotropic response of the system could be modeled within the system matrix of the iterative reconstruction algorithm. 


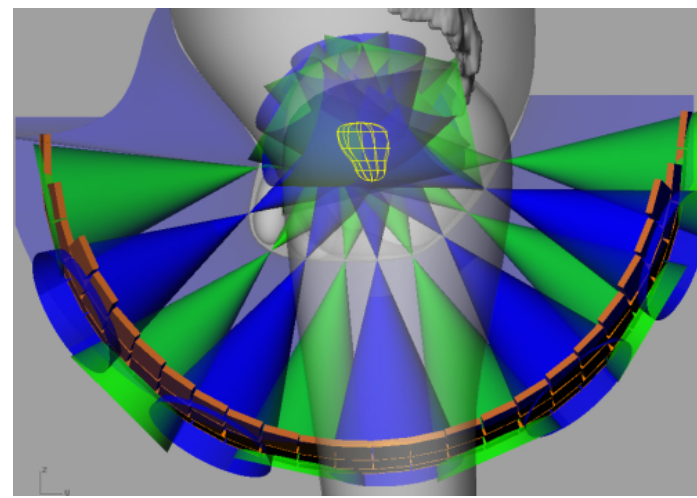

(a)

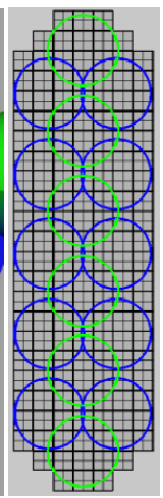

(b)

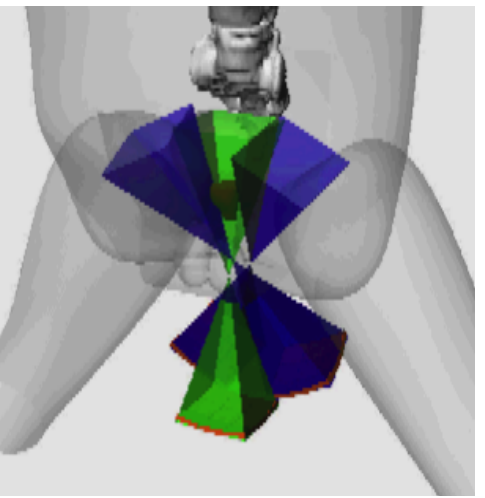

(c)

Figure 12. (a) Sixteen pinhole configuration for imaging the prostate and pelvic region. (b) Blue and green circles depict the extent of multiplexing for a circular aperture. (c) Square aperture from secondary shield reduces multiplexing and maximizes fill factor (right).

Focal imaging of the system is demonstrated by the phantom source reconstructions. Even with a uniform uptake of activity within the prostate and out of field activity from the bladder, a localized tumor may be detected. Registration of these images with transrectal ultrasound or a MRI based system ${ }^{50}$ may improve prostate biopsy. Image fusion may be implemented using the urethra as an anatomical landmark.

\section{CONCLUSIONS}

The design and simulation of a novel SPECT system for molecular imaging of the prostate has been presented. Using a morphological based collimator and tiled CZT detectors, the source to collimator distance can be reduced greatly compared to conventional SPECT systems. Monte Carlo transport simulations demonstrate the system's relative high spatial resolution and sensitivity. In addition, the system has the capability to provide focal spot imaging. These images may help in the guidance of biopsies.

\section{REFERENCES}

[1] American Cancer Society, "Cancer Facts and Figures 2010", Atlanta, GA, www.cancer.org

[2] Andriole, G. L., Bullock, T. L., Belani, J. S., Traxel, E., Yan, Y., Bostwick, D. G. and Humphrey, P. A., "Is there a better way to biopsy the prostate? Prospects for a novel transrectal systematic biopsy approach," Urology 70, 22-26 (2007).

[3] Zaheer, A., Cho, S. Y. and Pomper M. G., "New agents and techniques for imaging prostate cancer," J. Nucl. Med. 50, 1387-1390 (2009).

[4] Kelloff, G. J., Choyke, P. and Coffey, D. S., "Challenges in clinical prostate cancer: role of imaging," American Journal of Radiology 192, 1455-1470 (2009).

[5] Turkbey, B., Pinto, P. A. and Choyke, P. L., "Imaging techniques for prostate cancer: implications for focal therapy," Nature Reviews Urology 6, 191-203 (2009).

[6] Taneja, S. S., "Prostate Biopsy: Targeting cancer for detection and therapy," Rev Urology 8(4), 173-182 (2006).

[7] Jadvar, H., "Prostate cancer: PET with F-18-FDG, F-18 or C-11-Acetate, and F-18 or C-11-Choline," J. Nucl. Med. 52, 81-89 (2011).

[8] Jones, J. S., Patel, A., Schoenfield, L., Rabets, J. C., Zippe, C. D. and Magi-Galluzzi, C., "Saturation technique does not improve cancer detection as an initial prostate biopsy strategy," Journal of Urology 175, 485-488 (2006).

[9] Testa, C., Schiavina, R. and Lodi, R., "Prostate cancer: sextant localization with MR imaging, MR spectroscopy, and F-11-choline PET/CT," Radiology 244, 797-806 (2007). 
[10] Giovacchini, G., Picchio, M. and Coradeschi, E., "C-11-choline uptake with PET/CT for the initial diagnosis of prostate cancer: relation to PSA levels, tumor stage and anti-androgenic therapy," European Journal of Nuclear Medicine Molecular Imaging 35, 1065-1073 (2008).

[11] Pomper, M. G., Musachio, J. L., Zhang, J., Scheffel, U., Zhou, Y., Hilton, J., Maini, A., Dannals, R. F., Wong, D. F. and Kozikowski, A. P., "11C-MCG: synthesis, uptake selectivity, and primate PET of a probe for glutamate carboxypeptidase II (NAALADase)," Molecular Imaging 1, 96-101 (2002).

[12]Foss, C. A., Mease, R. C., Fan, H., Wang, Y., Ravert, H. T., Dannals, R. F., Olszewski, R., Heston, W. D., Kozikowski, A. P. and Pomper, M. G., "Radiolabeled small molecule ligands for prostate-specific membrane antigen: in vivo imaging in experimental models of prostate cancer," Clinical Cancer Research 11, 4022-4028 (2005).

[13] Guilarte, T. R., McGlothan, J. L., Foss, C. A., Zhou, J., Heston, W. D., Kozikowski, A. P. and Pomper, M. G., "Glutamate carboypeptidase II levels in rodent brain using [125I]DCIT quantitative autoradiography," Neuroscience Letters 387, 141-144 (2005).

[14]Zhou, J., Neale, J. H., Pomper, M. G. and Kozikowski, A. P., "NAAG peptidase inhibitors and their potential for diagnosis and therapy," Nat Rev Drug Discov 4, 1015-1026 (2005).

[15] Banerjee, S. R., Foss, C. A. and Castanares, M., "Synthesis and evaluation of technetuim-99m- and rhenium-labeled inhibitors of the prostate-specific membrane antigen (PSMA)," Journal of Medicinal Chemistry 51, 4504-4517 (2008).

[16] Barinka, C., Byun, Y., Dusich, C. L., Banerjee, S. R., Chen, Y., Castanares, M., Kozikowski, A. P., Mease, R. C., Pomper, M. G. and Lubkowski, J., "Interactions between human glutamate carboxypeptidase II and urea-based inhibitors: structural characterization," Journal of Medicinal Chemistry 51, 7737-7743 (2008).

[17] Chen, Y., Foss, C. A., Byun, Y., Nimmagadda, S., Pullambhatla, M., Fox, J. J., Castanares, M., Lupold, S., Babich, J., Mease, R. C. and Pomper, M. G., "Radiohalogenated prostate-specific membrane antigen (PSMA)-based ureas as imaging agents for prostate cancer," Journal of Medicinal Chemistry 51, 7933-7943 (2008).

[18] Chandran, S. S., Banerjee, S. R., Mease, R. C., Pomper, M. G. and Denmeade, S. R., "Characterization of a targeted nanoparticle functionalized with a urea-based inhibitor of prostate-specific membrane antigen (PSMA)," Cancer Biol Ther 7, 974-982 (2008).

[19] Hillier, S. M., Maresca, K. P., Femia, F. J., Marquis, J. C., Foss, C. A., Nguyen, N., Zimmerman, C. N., Barrett, J. A., Eckelman, W. C., Pomper, M. G., Joyal, J. L. and Babich, J., "Preclinical evaluation of novel glutamate-urealysine analogues that target prostate specific membrane antigen as molecular imaging pharmaceuticals for prostate cancer," Cancer Research 69, 6932-6940 (2009).

[20]Chen, Y., Dhara, S., Banerjee, S. R., Byun, Y., Pullambhatla, M., Mease, R. C. and Pomper, M. G., "A low molecular weight PSMA-based fluorescent imaging agent for cancer," Biochemical And Biophysical Research Communications 390, 624-629 (2009).

[21] Nargund, V., Al Hashmi, D. and Kumar, P., "Imaging with radiolabelled monoclonal antibody (MUJ591) to prostate-specific membrane antigen in staging of clinically localized prostatic carcinoma: comparison with clinical, surgical and histological staging," British Journal of Urology 95, 1232-1236 (2005).

[22] Kularatne, S. A., Zhou, Z., Yang, J., Post, C. B. and Low, P. S., "Design, synthesis, and preclinical evaluation of prostate-specific membrane antigen targeted Tc-99m-radioimaging agents," Mol Pharm 6, 790-800 (2009).

[23] Barrett, J. A., LaFrance, N. D. and Coleman, R. E., "Targeting metastatic prostate cancer in patients with I-123MIP1072 and I-123-MIP1095," J. Nucl. Med. 50(suppl), 136P (2009).

[24] Beekman, F. J., van der Have, F., Vastenhouw, B., van der Linden, A., Van Rijk, P. P., Burbach, J. P. and Smidt, M. P., "U-SPECT-I: A novel system for submillimeter-resolution tomography with radiolabeled molecules in mice," J. Nucl. Med. 46, 1194-1200 (2005).

[25] Cao, Z., Bal, G., Accorsi, R. and Acton, P. D., "Optimal number of pinholes in multi-pinhole SPECT for mouse brain imaging-a simulation study," Physics in Medicine \& Biology 50, 4609-4624 (2005).

[26] Furenlid, L. R., Wilson, D. W., Chen, Y., Kim, H., Pietraski, P. J., Crawford, M. J. and Barrett, H., "FastSPECT II: A second-generation high-resolution dynamic SPECT imager," IEEE Trans. Nucl. Sci. 51, 631-635 (2004).

[27] Gross, K., Kupinski, M. A., Peterson, T. and Clarkson, E., "Optimizing a multiple-pinhole SPECT system using the ideal observer," Proc. SPIE 5034, 314-322 (2003).

[28] Jorgensen, A. K. and Zeng, G. L., "Evaluation of multiplexing in multi-pinhole SPECT," 2005 IEEE Nuclear Science Symposium Conference Record, 2213-2217 (2005).

[29] Lackas, C., Schramm, N. U., Engeland, U. and Halling, H., "T-SPECT: A novel imaging technique for small animal research,” IEEE Nuclear Science Symposium Conference Record, 1842-1844 (2004). 
[30] Min, B. J., Choi, Y., Lee, N., Lee, K., Ahn, Y. C. and Joung, J., "Design consideration of a multipinhole collimator with septa for ultra high-resolution silicon drift detector modules," Nuclear Instruments \& Methods 606, 755-761 (2009).

[31] Peterson, T. E., Shokouhi, S., Furenlid, L. R. and Wilson, D. W., "Multi-pinhole SPECT imaging with silicon strip detectors," IEEE Nuclear Science Symposium Conference Record, 2752-2756 (2005).

[32] Wilson, D. W., Barrett, H. H. and Clarkson, E. W., "Reconstruction of two- and three-dimensional images from synthetic-collimator data," IEEE Transactions in Medical Imaging 19, 412-422 (2000).

[33] Schramm, N. U., Ebel, G., Engeland, U., Schurrat, T., Behe, M. and Behr, T. M., "High-resolution SPECT using multipinhole collimation," IEEE Trans. Nucl. Sci. 50, 315-320 (2003).

[34] Smith, M. F., Meikle, S. R., Majewski, S. and Weisenberger, A. G., "Design of multipinhole collimators for small animal SPECT,” IEEE Nuclear Science Symposium Conference Record, 2291-2295 (2004).

[35]Zimmerman, R. E., Moore, S. C. and Mahmood, A., "Performance of a triple-detector, multiple-pinhole SPECT system with iodine and indium isotopes," IEEE Nuclear Science Symposium Conference Record, 2427-2429 (2004).

[36] Metzler, S. D. and Jaszczak, R. J., "Simultaneous multi-head calibration for pinhole SPECT," IEEE Trans. Nucl. Sci. 53, 113-120 (2006).

[37]Funk, T., Kirch, D. L., Koss, J. E., Botvinick, E. H. and Hasegawa, B. H., "A novel approach to multipinhole SPECT for myocardial perfusion imaging," J. Nucl. Med. 47, 595-602 (2006).

[38] Kim, H., Furenlid, L. R., Crawford, M. J., Wilson, D. W., Barber, H. B., Peterson, T. E., Hunter, W. C. J., Liu, Z., Woolfenden, J. M. and Barrett, H.H., "SemiSPECT: a small-animal SPECT imager based on eight CZT detector arrays," Med. Phys., 33(2), 465-474 (2006).

[39] Tungsten Heavy Powder, Inc. (THP), www.tungstenheavypowder.com

[40] Miller, B. W., Moore, J. W., Gehm, M. E., Furenlid, L. R. and Barrett, H. H., "Novel applications of rapid prototyping in gamma-ray and x-ray imaging," IEEE Nuclear Science Symposium Conf. Record, 3322-3326 (2009).

[41] Wienmann, A. L, Hruska, C. B., O'Connor, M. K., "Design of optimal collimation for dedicated molecular breast imaging sytems," Med Phys 36(3), 845-856 (2009).

[42] Gamma Medica, Inc. (GMI), www.gammamedica.com

[43] Robert McNeel and Associates, www.rhino3d.com

[44] Barrett, H. H. and Swindell, W., [Radiological Imaging: The Theory of Image Formation, Detection, and Processing], Academic Press, New York, Vol. 1. (1981).

[45] Cherry, S. R., Sorenson, J. A. and Phelps, M. E, [Physics in Nuclear Medicine], Saunders, (2003).

[46] Segars, W. P., Lalush, D. S. and Tsui, B. M. W., "A realistic spline-based dynamic heart phantom." IEEE Trans. Nucl. Sci. 46(3), 503-506 (1999).

[47] Lee, C., Lodwick, D., Hurtado, J., Pafundi, D., Williams, J. L. and Bolch, W. E., "The UF family of reference hybrid phantoms for computational radiation dosimetry," Physics in Medicine \& Biology 55, 339-363 (2010).

[48] Brown, F. B., "MCNP Version 5," Transactions of the American Nuclear Society 87, 273 (2002).

[49] McNeal, J. E., Redwine, E. A., Freiha, F. S. and Stamey, T. A., "Zonal distribution of prostatic adenocarcinoma. Correlation with histologic pattern and direction of spread," Am J Surg Pathol 12(12), 897-906 (1988).

[50] Song, S., Cho N. B., Tokuda, J., Hata, N., Tempany, C. M., Fichtinger, G. and Iordachita, I., "MRI compatibility study of a pneumatically actuated robotic system for transperineal prostate needle placement," CARS 2010 Proc. $24^{\text {th }}$ Int. Con. And Exhibition, Int J CARS 5(Suppl 1), 26-27 (2010). 